\title{
New capabilities for Monte Carlo simulation of deuteron transport and secondary products generation
}

\author{
P. Sauvan , J. Sanz, F. Ogando \\ Departamento de Ingeniería Energética, E.T.S.Ingenieros Industriales, UNED, C/Juan del Rosal i2, 28040 Madrid, Spain
}

\begin{abstract}
A B S T R A C T
Several important research programs are dedicated to the development of facilities based on deuteron accelerators. In designing these facilities, the definition of a validated computational approach able to simulate deuteron transport and evaluate deuteron interactions and production of secondary particles with acceptable precision is a very important issue. Current Monte Carlo codes, such as MCNPX or PHITS, when applied for deuteron transport calculations use built-in semi-analytical models to describe deuteron interactions. These models are found unreliable in predicting neutron and photon generated by low energy deuterons, typically present in those facilities.

We present a new computational tool, resulting from an extension of the MCNPX code, which improve significantly the treatment of problems where any secondary product (neutrons, photons, tritons, etc.) generated by low energy deuterons reactions could play a major role. Firstly, it handles deuteron evaluated data libraries, which allow describing better low deuteron energy interactions. Secondly, it includes a reduction variance technique for production of secondary particles by charged particle-induced nuclear interactions, which allow reducing drastically the computing time needed in transport and nuclear response calculations. Verification of the computational tool is successfully achieved. This tool can be very helpful in addressing design issues such as selection of the dedicated neutron production target and accelerator radioprotection analysis. It can be also helpful to test the deuteron cross-sections under development in the frame of different international nuclear data programs.
\end{abstract}

Keywords:

MCNPX

Monte Carlo

Cliarged particles

Transport library

Variance reduction

\section{Introduction}

Several important research programs, based on using a deuteron accelerator facility, are currently under development. Some of the most important are SARAF [1], SPIRAL2 [2| and IFMIF/ EVEDA [3,4], in which deuterons of relatively low energy (less than $40 \mathrm{MeV}$ ) interact with a dedicated target to produce neutrons for different applications. In IFMIF/EVEDA facility the major application is to simulate the radiation damage generated in the materials of a nuclear fusion reactor. Other facilities, addressing also the simulation of fusion reactor materials, follow a complementary approach by using triple ion beams, in which one of them can be a deuteron beam. A recent proposal at this respect is the Spanish Technofusión Project [5].

In designing these facilities, the definition of a validated computational approach, able to simulate deuteron transport and evaluate deuteron interactions and production of secondary particles with acceptable precision, is a very important issue.
Some important applications of the methodology are related to the selection of the dedicated neutron production target and the accelerator radioprotection analysis.

Current Monte Carlo codes, such as MCNPX [6] or PHITS [7], when applied for deuteron transport calculations use built-in semi-analytical models to describe deuteron interactions. For the energy physics domain corresponding to the deuterons typically present in those facilities (lower than $40 \mathrm{MeV}$ ), these models are found unreliable in predicting neutron and photon generated by these low energy deuterons [8-11].

These codes were tested regarding their capability to predict neutron production for EVEDA conditions for $\mathrm{Cu}[10]$, as beam stop material, as well as for other typical elements of accelerator equipments [11] in an energy range from 0 to $20 \mathrm{MeV}$. Crosssections for neutron produced by deuteron reactions were calculated with the different nuclear models included in MCNPX and PHITS and compared with available experimental data. Results exhibited a bad fit with experimental values, and showed a very unrealistic performance in the very low energy range below $5 \mathrm{MeV}$ - showing that the neutron production was inexistent for most of the models or very low for the rest. In addition, models were found to give unrealistic spectrum of the 
emitted neutrons, showing a significant contribution of an unphysical high energy tail.

In the frame of the JFMIF facility, a MCNPX deficient performance has been also reported to predict neutron yields due to interactions of deuterons in a thick lithium target [12|. For the same application, a similar conclusion was reported for PHITS [13].

In this context, a new computational methodological approach was proposed [10] based on the use of an extended MCNPX version capable to use evaluated deuteron libraries for neutron (and gamma) production. The Talys nuclear reaction code [14] was the first option suggested $[10,11]$ to be explored as potentia] candidate to produce the evaluated data for double-differential neutron and photon emission cross-sections for incident deuterons up $40 \mathrm{MeV}$. The potential of Talys to produce deuteron evaluated data files for neutron production with enough quality has been explored in the range from 0 to $20 \mathrm{MeV}$ for several elements of interest in the EVEDA prototype accelerator $[8,9]$. The conclusion was that utilization of Talys, with appropriate adjusting parameters in some cases, produce deuteron crosssections showing a good reproduction of experimental data and providing consistent spectrum for the emitted neutrons.

Up to now, there was not general transport cross-section libraries for charged particles (except proton LA150H [15| for MCNPX); due partially to the fact that even today there are not Monte Carlo transport codes (except MCNPX for protons) able to handle them. Currently, this situation is changing, and recently, the first deuteron cross-section data set for transport calculations has been produced, as pait of TENDL-2008 (Talys Evaluated Nuclear Data Library). A TENDL-2009 version is expected to be released at the end of 2009 . The TENDL library [16], generated with Talys code, provides nuclear data for different incident light charged particles (including deuteron) interacting with various elements. The developinent of a complete deuteron transport libracy is a task recently included in some of the important nuclear data development projects. In the JEFF project this transport libracy is scheduled to be included as a section in the general purpose libraly called JEFF-3.2, and in the frame of the FENDL Program is going to be included as pait FENDL-3. In this context, it is fully justified to extend Monte Carlo transport codes with the capability to handle deuteron evaluated files. The presentation of the aspects related with the implementation and verification of this capability is one of the objectives of this paper.

Another impoitant drawback of current Monte Carlo codes when applied to transport of low-energy light jons, and associated secondary products and nuclear responses calculation, is the huge amount of computing time that can be required to address some important problems. This will take place in problems where one or several types of secondary products plays a major role. In these problems a large number of primary source particles is needed to produce a significant amount of secondary particles because the nuclear interaction probability of low energy light ions with material is low. A good example is the calculation of prompt dose rates outside the accelerator vault. The responsible for these doses are the neutrons and photons produced due to the fact that deuterons will interact (i) with materials all along the beam line where deuterons are lost, and (ii) at the end of the beam line, in the bean dump where the whole beam is stopped during the testing phase, or in the dedicated target in the normal operational phase. In the assessment of the dose, a large amount of deuteron histories will be needed in order to generate enough secondary neutrons to have good statistics in the dose rate evaluation. At low energy, ions have short track lengths inside the material due to the slowing down of the particle. This short track associated with a relatively low absorption cross-section, leads to a very low production of secondary particles. This feature of low-energy light ion transport means to waste a lot of computing time in transporting primary particles that have no contribution in the calculation of the response functions associated to the production and transport of secondary particles. It is worthwhile mentioning than in addition to the neutron and gamma secondary radiation, the production and transport of other secondary products, such as tritons [17], should be also taken into account for different purposes.

We will present in this paper the features of the MCUNED code developed as an MCNPX extension to deal with problems where any secondary product (neutrons, photons, tritons, etc.) generated by deuterons induced nuclear reactions could play a major role. In Section 2 the new capabilities implemented in MCNPX are presented: (i) the capability to handle deuteron data libraries (residual production cross-sections and energy-angle distributions of all outgoing particles); and (ii) special emphasis is devoted to explain the basis of the variance reduction technique proposed to be used in charged particle transport calculations. It is worth mentioning that since MCUNED is derived from MCNPX (they have the same code core), the MCUNED input is fully compatible with any MCNPX input, and its transport techniques are as reliable as in the original code. Likewise, MCUNED keep all the MCNPX original capabilities, such as, flexibility in definition of the charged particle source, in the definition of the geometry of the system, ptrac option, nuclear responses, etc. The performing of all of them has not experienced any variation with the implementation of the new capabilities. In Section 3 the verification activities of the new capabilities implemented in the extended MCNPX version (MCUNED code) over the original one is presented: first, the verification of the correct reading of deuteron libraries is assessed; secondly, the results of the tests selected for the verification process of the variance reduction technique are assessed. All the capabilities of the original MCNPX are available in the MCUNED version and have been tested to work properly. In the last section, conclusions emphasis the improvement that the new developments means to deal with problems related to deuteron transport, and associated secondary particle production and nuclear and radiological response functions.

\section{Methodology}

\subsection{Handling with deuteron libraries}

The MCNPX subroutines devoted to read and process proton nuclear cross-section from libraries have been modified and extended allowing the use of deuteron libraries. Subroutines dedicated to the sample of nuclear reactions have been as well modified in order to use tables instead of models during the deuteron transport. The charged particle transport process remains the original from MCNPX.

\subsection{Variance reductian technique far light jons}

In radioprotection studies, most of the shielding challenges come from neutral particles like neutron and photon. In accelerator radioprotection studies, neutrons and photons are produced by nuclear reactions between accelerated jons and the accelerator materials. Hence, the determination of the neutrals particle source, which are secondary particles, requires the accurate transpoit of the primaty ions.

When the interest of the simulation is emphasized in the transport of secondary particle, it is necessary to perform the calculation with a large number of secondary particles histories in 
order to reduce the statistical error. Moreover, the secondary particles source has to be distributed adequately (energetically and spatially) with respect to the simulated primary particle source. The problem arises when the production of secondary per primary particles (s/p) is very low. In this case, it is necessary to consider $(s / p)^{-1}$ times more primary particles histories to obtain the desired number of secondary particles.

If primary particles are low energy ions, the problem highlighted above takes a real importance. Due to the slowing down of the ions, these have a short track length (about few hundred microns) and a quick loss of energy. For a reaction cross-section about $1 \mathrm{~b}$ and a typical material atomic density about $10^{23} \mathrm{~cm}^{-3}$, the order of magnitude of the ratio (s/p) will be $10^{-5}$.

The main idea of the new variance reduction technique implemented in MCUNED, for the production of secondary particles from nuclear reaction with charged particle, is to always force the nuclear reaction, taking care of the correct transport of charged particle, giving rise to the absorption of the incident particle by the target nucleus, for each charged particle history. The weight of the secondary particle produced is then multiplied by the probability of such nuclear absorption, in order to keep the yield of secondary particle production. This variance reduction technique is valid for all kind of ion, but it is not suitable for electron or positron because it does not take into account bremsstrahlung effect which is negligible for ions.

The procedure employed in this technique is to record the track of the particle for each ion history. This record contains for each location along the track, the position $\vec{R}_{i j}$ (where $i$ is the history index and $j$ the index referring to the particle location along the trajectory), the energy $E_{i, j}$ and the direction $\vec{\Omega}_{i, j}$ of the ion along its track (Fig. 1). The particle transport is performed by the dedicated MCNPX subroutine in which the nuclear absorption reactions have been disabled. This means that the ion transport is carried out taking into account only energy-loss straggling and nuclear elastic scattering processes. The ion track is recorded until the energy of the particle falls below a given energy cutoff or enter into a cell of zero importance, then the particle history terminates. With this track, one can evaluate the probability density function $P(I)$ of the ion nuclear absorption into the material according to the absorption cross-section

$P(l)=\exp \left(-\int_{0}^{l} \rho(s) \sigma_{a}(E(s)) d s\right) \rho(l) \sigma_{a}(E(l))$

where $\rho(l)$ and $E(l)$ are the material atomic density and the energy of the particle at the location I; $\sigma_{a}$ the absorption cross-section.

With the probability density function $P(I)$ of the charged particle one can sample the location of the different nuclear absorption reactions. Each event is then produced at a different location with a probability density for a particular position given by $P(l)$

Once the position of the event is sampled, one can retrieve for this position the corresponding particle energy and direction. All the incident jon parameters are then known to sample the production of the secondary particle. The number of type $k$ particle produced for each absorption is given by $\sigma_{k}(E) / \sigma_{a}(E)$ (where $\sigma_{k}(E)$ is the particle production cross-section).

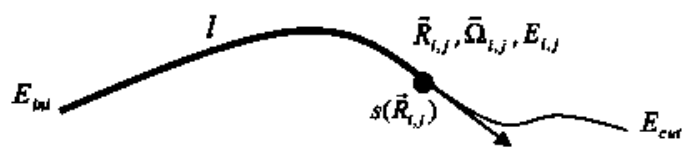

Fig. 1. Track of a given ion history i. This trajectory take into account only scattering, no nuclear absorption processes are taken into account. The particle is killed when its energy fall below the energy cutof $E_{\text {cut }}$ or enter a cell of zero importance.
Finally, the weight of the secondary particles is normalized with the value of the absorption probability $P_{a}=\int_{0}^{L_{1}} P(l) d$ l (being $L_{i}$ the total length of the history track $j$ ), and transported with the unmodified MCNPX subroutines. Obviously, for each source ion history the trajectory will be different.

This same idea is implemented in the MCDelicious code [12], with the distinction that in the MCDelicious case the trajectories of deuterons are rectilinear.

The implementation of such variance reduction technique allows saving a large amount of computing time with respect to normal execution of MCNPX. The time used with or without variance reduction can be estimated. Taking $T_{p}$ as the time spend to calculate one primaty particle history and $T_{s}$ the time necessaty for transporting the secondary particles produced by the source particle nuclear reaction, the time needed to produce and transport secondary particles without variance reduction will be $T_{\text {without }} \approx v T_{p}+T_{s}$ (with $v=(s / p)^{-1}$ ), while with variance reduction the new time is $T_{V R} \approx T_{p}+T_{s}$. The time saving gain is then

$G=\frac{T_{\text {without }}}{T_{V R}}=\left(\frac{v+\left(T_{s} / T_{p}\right)}{1+\left(T_{s} / T_{p}\right)}\right)$

Since, for low energy, the number of secondary particles per source particle is below $10^{-2}$ (and sometime several orders of magnitudes lower), if $T_{s}$ and $T_{p}$ are of the same order of magnitude, the gain will be about 100 .

This technique is more efficient when the number of secondary particles per source particle is low. In some case, for example for tritium production (which production can be about $10^{-8}$ tritium per charged particles), this technique is the only means of getting reliable results on the production of secondary particles.

\section{Verification activities}

\subsection{Hondling of deuteron tibrories for transport calculation: implementation in MCPNX}

This section is devoted to check that the modification of MCNPX subroutine that allows reading deuteron libraries is coirect. To cairy out this verification, neutron spectra emitted from thin Copper-63 target interacting with $9 \mathrm{MeV}$ deuteron beam have been recorded at different angles. The use of a thin target ensures that deuteron reactions will take place with little or no previous slowing down. By this way, reaction cross-section may be directly compared with existing tables. The MCUNED spectra have been converted in neutron double differential cross-section and compared with cross-section supplied by ENDF format library.

The deuteron library used in this simulation is provided by the Tendl-2008 package. For all isotopes present in the database, this package supply libraries in both ENDF and ACE format. The ACE format library is used directly in transport calculation while the ENDF format library is processed with Janis 3.0 software to obtain the double differential cross-section that will be used as reference. Since the MCUNED results are in the laboratory frame, the cross-sections given by Janis 3.0 have been transformed from the center-of-mass system to laboratory system.

The first confirmation of the correct management of the deuteron library by MCUNED is the value of the neutron yield and the total neutron cross-section given by MCUNED. In this simulation, thanks to the reduced thickness of the target, the energy of charged particle can be supposed constant, and its trajectory a straight line of length I (I being the target thickness). With these assumptions, the neutron yield can be easily obtained using the variance reduction technique. Since this technique produces one absorption reaction per history, the neutron yield $\sigma_{n} / \sigma_{a}$ (where $\sigma_{n}$ is the neutron production cross-section and 
$\sigma_{a}$ the absorption cross-section) is merely the number of created neutron divided by the history number. The total neutron crosssection is obtained by the relation $n / d-\rho \sigma_{\text {II }}$ where all the parameters except the cross-section are known. The following table shows the result of the neutron cross-section and neutron yield given by MCUNED and the corresponding values extracted from the ENDF library.

The Fig. 2 shows the neutron emission double differential cross-section obtained from ENDF format libraty and the crosssection calculated with MCUNED for 4 different angles.

Both integrated values (neutron yield and total cross-section) and double differential cross-section given by MCUNED are matching those present in the table. This result validates the management of deuteron cross-section tables in MCUNED (Table 1).

\subsection{Verification of the variance reductian technique}

The verification of the variance reduction technique is carried out running proton transport simulation with the original MCNPX code and with MCUNED code. These comparisons will emphasize the great advantage in using the variance reduction technique. Two tests are performed: the first one is devoted to check the ability of the variance reduction technique to reproduce neutron production, flux and spectrum in thick target. The second one verifies that the response function like neutrons and photons dose is also valid. This last one would validate implicitly the correct photon production and flux calculations.

The first test consists in impacting a $10 \mathrm{MeV}$ proton beam onto a cylindrical copper taiget, $1 \mathrm{~m}$.n thickness and $1 \mathrm{~cm}$ radius, recording the neutron spectium at different angles respect to the beam axis. The number of histories introduced in each simulation has been chosen to produce the same quantity of secondary neutrons histories, in order to reach the same statistical error in both simulations.

In Table 2 the number of histories used and results from both simulations are displayed to exhibit the benefit in using the variance reduction technique. In the MCNPX calculation, the number of neutron per proton is the number of produced neutron histories divided by the number of histories. In the MCUNED calculation this value cannot be evaluate in the same way because the variance reduction technique produce one absorption per history and the produced particle is weighted by the absorption probability.

Figs. 3-5 show the comparison between MCNPX and MCUNED of the neutron energy spectra and the neutron angular distribution. In all these figures the relative error between both computations is lower than 1\% (except in minor number of points where the statistical error is high). The good agreement between both simulations traduces the correct implementation of the variance reduction technique into the MCUNED code. The reduction in computing time is in this case outstanding, being the time needed by MCNPX three orders of magnitude higher than with MCUNED simulation.

The last test is the calculation of the neutron and photon ambient equivalent dose produced by a $100 \mathrm{~mA}$ proton beam at $6.7 \mathrm{MeV}$ on a cylindrical nickel thick target. This simulation has the characteristics parameters of the beam-stop itradiation condition of the LEDA accelerator [18]. This simulation setup

Table 1

Neutron yield and cross-section obtained from MCUNED simulation and given by ENDF data table.

\begin{tabular}{lll}
\hline & $\begin{array}{l}\text { Neutron yield per } \\
\text { absorption }\end{array}$ & $\begin{array}{l}\text { Neutron production } \\
\text { cooss-section (b) }\end{array}$ \\
\hline MCUNED & $\mathbf{0 . 8 4 2 8}$ & 0.7856 \\
ENDF & $\mathbf{0 . 8 4 3 0}$ & 0.7851 \\
\hline
\end{tabular}

Table 2

MCNPX-MCUNED performance comparison of neutron emitted from copper thick target. The computing time is the time necessary to perform the whole simulation with one processor computer.

\begin{tabular}{lll}
\hline & MCNPX & MCUNED \\
\hline Number of histories & $1 \times 10^{10}$ & $1 \times 10^{7}$ \\
Computing time & $1.37 \times 10^{5} \mathrm{~min}$ & $32 \mathrm{~min}$ \\
Produced neutron histories & $5.59 \times 10^{6}$ & $5.36 \times 10^{6}$ \\
Neutronf $/$ proton & $5.589 \times 10^{-4}$ & $5.587 \times 10^{-4}$ \\
Statistical error & $0.04 \%$ & $0.03 \%$ \\
\hline
\end{tabular}



Fig. 2. Neutron double diTerential cross-section from the reaction d-Cu63, with $9 \mathrm{MeV}$ deuteron. 
consists of a $100 \mathrm{~mA}$ proton current at $6.7 \mathrm{MeV}$ impacting a cylindrical nickel target of $1 \mathrm{~mm}$ thickness and $1 \mathrm{~cm}$ radius. The ambient equivalent dose produced by neutrons and photons have been evaluated. In order to obtain reference results a MCNPX computation of this simulation has been performed. The number of MCNPX histories has been selected in order to obtain a low statistical error for the neutron dose. This error value result of a deal between the computing time and a statistical error low enough to give reliability to the result used as reference.

Fig. 6 shows the neutron and photon dose map, computed by MCNPX, around the target. Each dose value plotted in Fig. 6 has a statistical error between $1 \%$ and $10 \%$ for neutron dose and between $0.2 \%$ and $2 \%$ for photon dose. The spatially averaged error is $4 \%$ for neutron dose and $0.8 \%$ for photon dose.

In order to compare MCUNED results, the statistical error of its calculation has to be in the same range as the statistical error of MCNPX calculation. The number of proton histories for MCUNED simulation has been set to get the same number of secondary particle histories than in MCNPX simulation, and reach the same statistical error as in MCNPX. For MCUNED calculation the statistical error in neutron and photon dose values lay in the

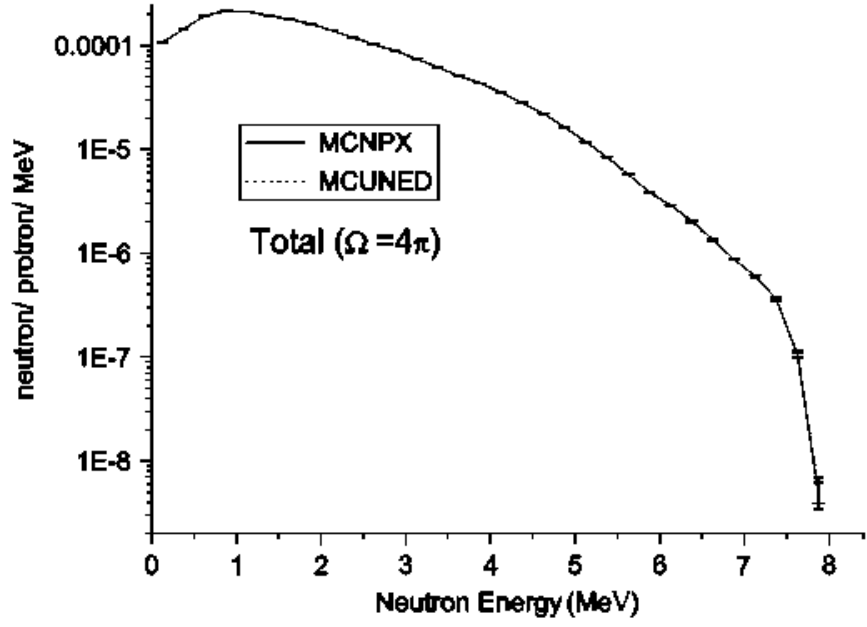

Fig. 3. 5pectral neutron yield from thick copper talget. same range than MCNPX errors. The spatially averaged error in both simulation is also very close each other.

The summary of the simulation is shown in Table 3.

To check the validity of MCUNED calculation the relative error

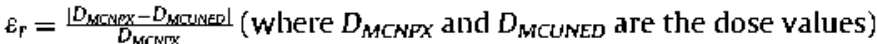
is introduced. A relative error $\varepsilon_{\text {, lying in the same range as }}$ statistical error would confirm a good agreement between both simulations.

The relative error map has been plotted in Fig. 7. These maps show the good agreement between both simulations; the relative error for neutron dose is below $10 \%$, with a spatially averaged error value of $4.3 \%$, and for photon dose below $2 \%$ with a spatially averaged error value of $1 \%$. These deferments spatially averaged error are summarized in Table 4.

In order to compare the results obtained by MCNPX and MCUNED code the relative error $\varepsilon_{r}=\frac{D_{\text {MCAPX }}-D_{\text {MCUNED }} \mid}{D_{\text {MAONPX }}}$, with $D_{\text {MCNPX }}$ and $D_{\text {MCUNED }}$ the dose calculated with MCNPX and MCUNED, respectively, between both simulation for neutron and photon dose have been evaluated. The results have been plotted in Fig. 7. These figures show the perfect agreement between both simulations;

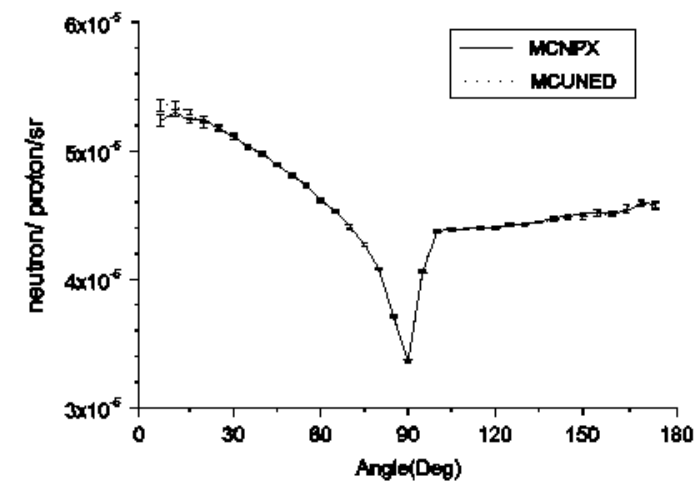

Fig 5. Energy integrated angular distribution of neutron yield from thick copper target. The dip at $90^{\prime \prime}$ come from the self-shielding of the target, neutrons emitted perpendicularly to the beam have a major path inside the target than those emitted in other directions.

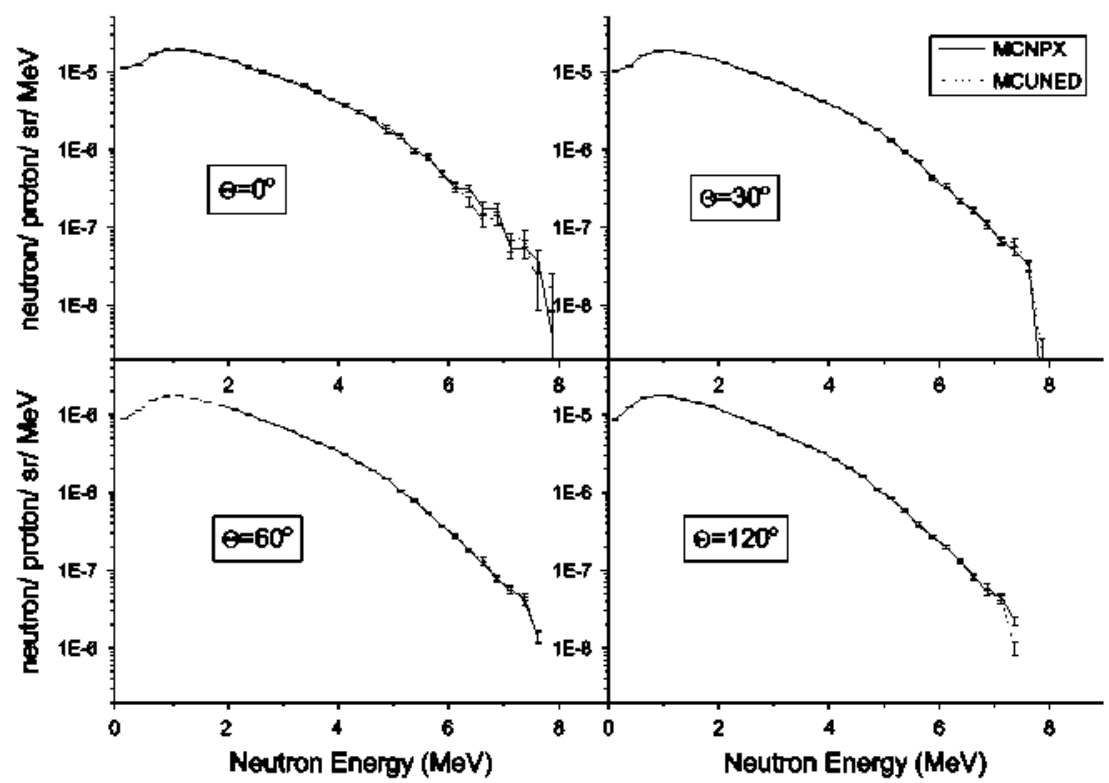

Fig 4. Neutron energy spectra at different emission angles from thick copper target. 

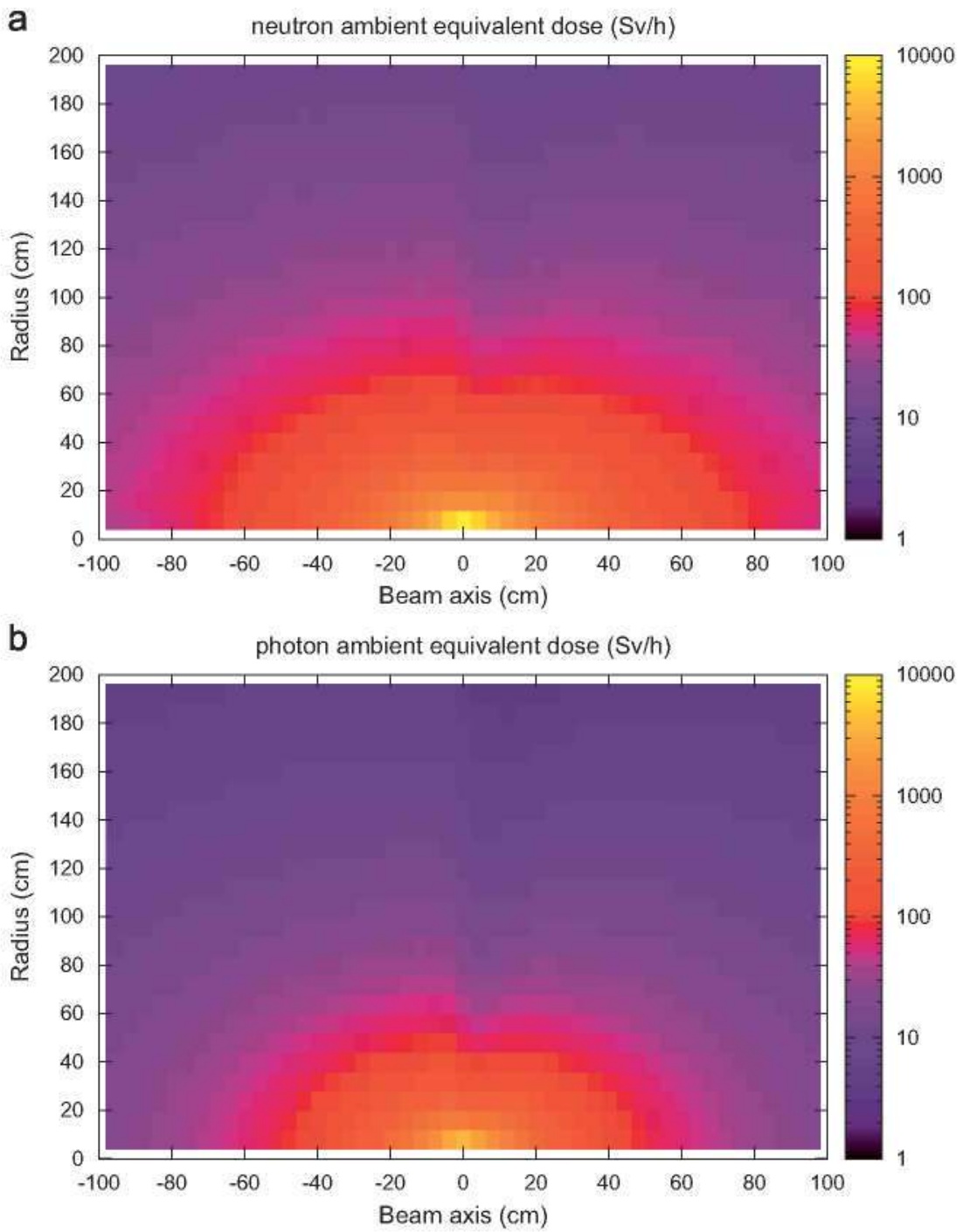

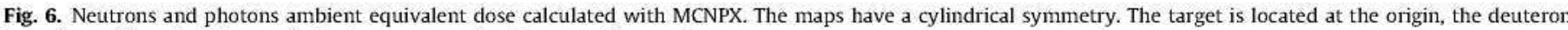
beam is positioned on the $z$ axis deuteron are coming from negative $z$ values.

Table 3

MCNPX-MCUNED performance comparison of the total neutron produced in the dose calculation. The computing time is the time necessary to perform the whole simulation with one processor computer.

\begin{tabular}{lll}
\hline & MCNPX & MCUNED \\
\hline Number of histories & $5 \times 10^{9}$ & $6.5 \times 10^{5}$ \\
Computing time & $6.2 \times 10^{4} \mathrm{~min}$ & $6 \mathrm{~min}$ \\
Produced neutron histories & $3.8 \times 10^{4}$ & $3.6 \times 10^{4}$ \\
Produced photon histories & $9.5 \times 10^{5}$ & $9.1 \times 10^{5}$ \\
\hline
\end{tabular}

the relative error is below $10 \%$ (with a most important number of relative error values around $2 \%$ ) for neutron dose and below $2 \%$ for photon dose. These relative errors depend on the statistical errors of each simulation.

\section{Conclusions}

We present two extensions to current Monte Carlo transport codes that allow dealing more efficiently with problems where any secondary product (neutrons, photons, tritons, etc.) generated by deuterons induced nuclear reactions could play a major role. The first refers to handling deuteron data libraries (residual production cross-sections and energy-angle distributions of all outgoing particles), which enables to include reliable nuclear cross-section in the deuteron transport processes. The second is a reduction variance technique for production of secondary products by charged particle-induced nuclear interactions. It is shown that this technique reduces drastically the number of ion source histories necessary to produce secondary products, and therefore reduces drastically the computing time needed in performing transport and nuclear response calculations with the target accuracy required in a given problem.

These two developments have been implemented into the MCNPX code. This extended MCNPX version (MCUNED Code) has been verified. The verification activities of the new two capabilities are presented and the results allow concluding that have been successfully verified. In this extended version all previouslyexisting MCNPX code capabilities have been fully preserved.

The extension of MCUNED to handle all kinds of light ions (proton, deuteron, triton, helium3, alpha) libraries is ongoing work. The interest to include the two mentioned capabilities in other Monte Carlo codes different than MCNPX is also under consideration. 

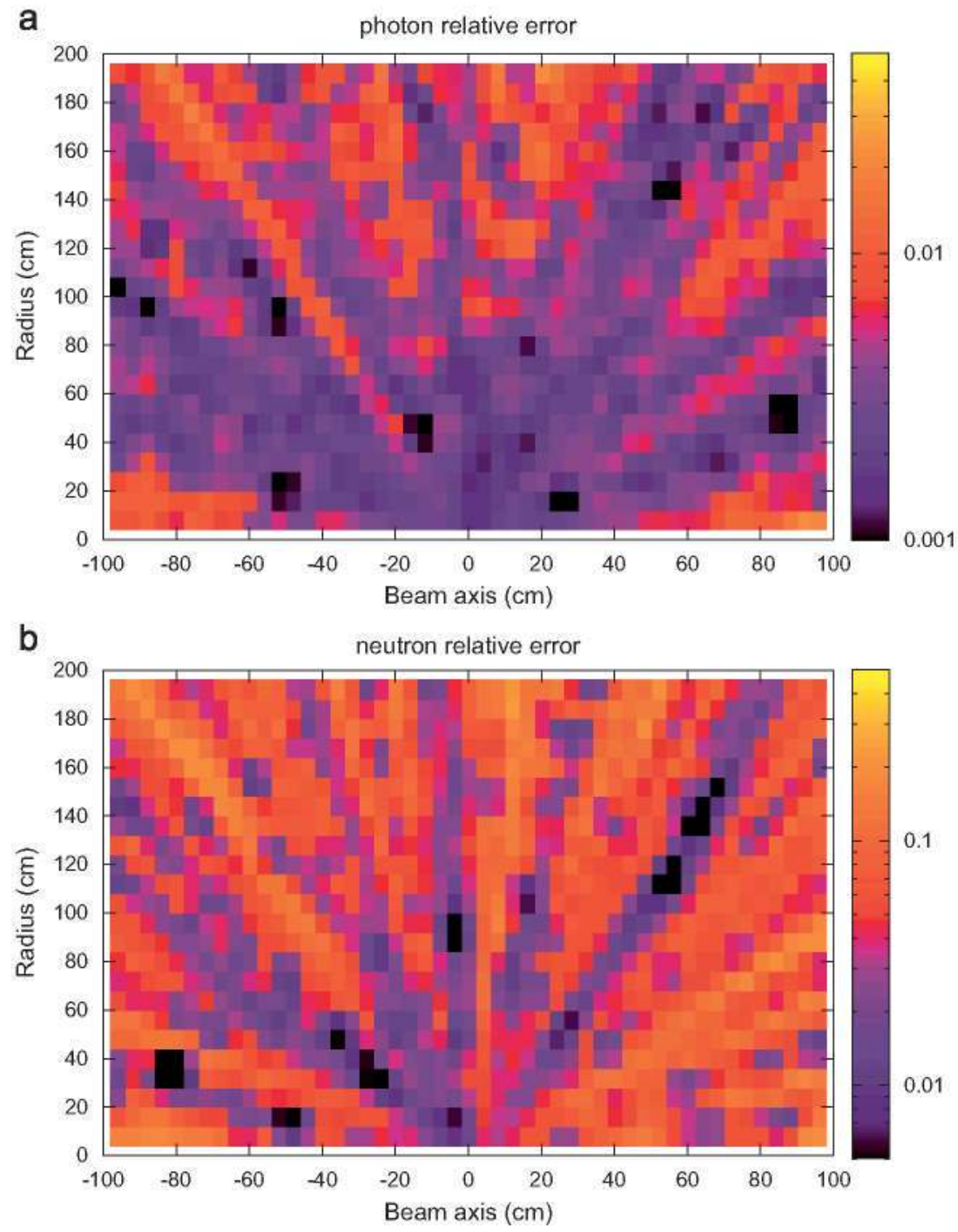

Fig. 7. Neutron and photon relative error between MCUNED and MCNPX calculations of the ambient equivalent dose shown in Fig. 6 .

Table 4

Spatially averaged error in neutron and photon dose calculations.

\begin{tabular}{lll}
\hline & Neutron (\%) & Photon (\%) \\
\hline MCNPX statistical error & 4.3 & 0.83 \\
MCUNED statistical error & 4.4 & 0.85 \\
MCNPX-MCUNED relative error $\varepsilon_{r}$ & 4.3 & 1.0 \\
\hline
\end{tabular}

\section{Acknowledgments}

This work has been supported by Association EURATOM/ CIEMAT for Fusion (AEC) and Plan Nacional de I+D+I (2008-2011) Fusion Nuclear, ENE2008-06403-C06-02, MCI, Spain.

\section{References}

[1] 1. Mardor, et al., Status of the SARAF CW $40 \mathrm{MeV}$ proton/deuteron accelerator, Particle Accelerator Conference, May 4-9, 2009, Vancouver, Canada, (FR5REP087).

[2] M. Lewitowicz, Acta Physica Polonica B 40 (2009) 811.

[3] IFMIF Comprehensive Design Report, by the IFMIF International Team, an activity of the International Energy Agency, Implementing Agreement for a Program of Research and Development on Fusion Materials, January 2004.

4] P. Garin, M. Sugimoto, Fusion Engineering and Design 84 (2009) 259-264

[5] J. Sánchez, et al., TechnoFusión: a new centre for the development of fusion technologies and fusion reactor devices, in: Proceeding of the 14th International Conference on Emerging Nuclear Energy Systems, June 29-July 3, 2009 Ericeira, Portugal.

[6] D.B. Pelowitz, ed.: MCNPX User's manual, Version 2.5.0, LA-CP-05-0369 (2005).

[7] K. Niita, H. Nakashima, T. Sato, H. Iwase, The present status of the PHITS code, Shielding Aspects of Accelerators, Targets and Irradiation Facilities 8th Meeting, Gyongbuk, Republic of Korea, 2006.

[8] J. Sanz, et al., Journal of Nuclear Materials 386-388 (2009) 991.

[9] M. García, et al., Nuclear Technology 168 (2009) 132.

[10] J. Sanz, et al., Fusion Science and Technology 56 (2009) 273.

[11] V. Blideanu, et al., Proceeding of the 14th International Conference on Fusion Reactor Materials, September 6-11, 2009, Sapporo, Japan.

[12] S.P. Simakov, U. Fischer, U. von Möllendorff, I. Schmuck, A.Yu. Konobeev, Yu.A. Korovin, P. Pereslavtsev, Journal of Nuclear Materials 307-311 (2002) 1710.

[13] D. Kaku, T. Yea, Y. Watanabe, in: O. Bersillon, F. Gunsing, E. Bauge, R. Jacqmin, S. Leray (Ed.), Proceedings of the International Conference on Nuclear Data for Science and Technology, April 22-27, 2007, Nice, France, EDP Sciences, 2008, pp. 987-990.

[14] A.J. Koning, S. Hilaire, M.C. Duijvestijn, in: O. Bersillon, F. Gunsing, E. Bauge, R. Jacqmin, S. Leray (Ed.), Proceedings of the International Conference on Nuclear Data for Science and Technology, April 22-27, 2007, Nice, France, EDP Sciences, 2008, pp. 211-214.

[15] M.B. Chadwick, et al., Nuclear Science and Engineering 131 (1999) 293. 
[15] A.J. Koning, S. Hilaire, M.C. Duijvestijn, (http:/(www.talys.eu/tendl-2008f).

[17] A. Mayoral, et al., Relevance of d-D interactions on neutron and tritium productions in IFMIF-EVEDA prototype, in: Proceeding of the 14th Intemational Conference on Fusion Reactor Materials, September 6-11, 2009, Sapporo. Japan.
[18] H. Vernon Smith, Jr., J. David Sclmeider, Status update on the low-energy demonstration accelerator (leda). LJNAC Conference Procedings (1998): T.H Van Hagan, D.W. Doll, J.D. Schneider, F.R. Spinos, Design of an ogive-shaped beamstop. LINAC Conference Proceedings (1998). 